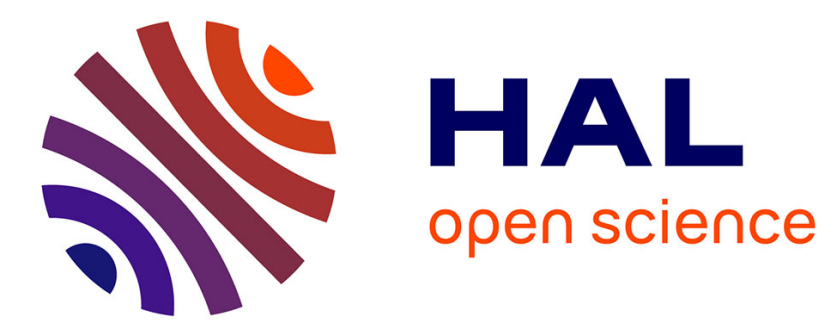

\title{
Urban Traffic Eco-Driving: Speed Advisory Tracking
} Giovanni de Nunzio, Carlos Canudas de Wit, Philippe Moulin

\section{To cite this version:}

Giovanni de Nunzio, Carlos Canudas de Wit, Philippe Moulin. Urban Traffic Eco-Driving: Speed Advisory Tracking. CDC 2014 - 53rd IEEE Conference on Decision and Control, Dec 2014, Los Angeles, United States. 10.1109/CDC.2014.7039651 . hal-01101280

\section{HAL Id: hal-01101280 \\ https://hal.univ-grenoble-alpes.fr/hal-01101280}

Submitted on 9 Jan 2015

HAL is a multi-disciplinary open access archive for the deposit and dissemination of scientific research documents, whether they are published or not. The documents may come from teaching and research institutions in France or abroad, or from public or private research centers.
L'archive ouverte pluridisciplinaire HAL, est destinée au dépôt et à la diffusion de documents scientifiques de niveau recherche, publiés ou non, émanant des établissements d'enseignement et de recherche français ou étrangers, des laboratoires publics ou privés. 


\title{
Urban Traffic Eco-Driving: Speed Advisory Tracking
}

\author{
Giovanni De Nunzio, Carlos Canudas de Wit, Philippe Moulin
}

\begin{abstract}
The evaluation of the benefits of an Eco-Driving assistance system is carried out in the urban environment in presence of traffic lights. The traffic evolution is modeled macroscopically with the Urban Variable-Length Model [1] in a variable speed limits framework. Under the assumption of equal boundary flows, the vehicles in a road section dispose themselves according to well defined equilibrium conditions which are dependent on the traveling speed in the free-flow part of the section. Given certain initial traffic conditions, an optimal speed limit for the section can be found in order to drive the system to an efficient equilibrium state. Further analysis of the equilibrium conditions and the stability properties of the system is conducted in this work. The system is proved to be controllable, under the working hypotheses, and a controller is designed to simulate the response of the drivers compliant to the eco-speed advisory. A sub-optimal control strategy is finally proposed also in the case of unequal boundary flows.
\end{abstract}

Index Terms - Traffic modeling, variable speed limits, optimal speed advisory, traffic lights.

\section{INTRODUCTION}

Research efforts have been focusing on the intelligence of transportation systems, and congestion control in the urban environment may be seen as mainly divided into supply-side and demand-side management.

On the supply side of the traffic control strategies, traffic lights timings optimization is one of the main congestionrelief measures. Very well known traffic-signals-timings optimization algorithms found wide application (TRANSYT, SCOOT, SCATS, TUC, etc.). In the last two decades traffic lights optimization strategies became more and more modelbased, and a variety of macroscopic models have been proposed or refined for control purposes [2][3][4][5][6].

The main philosophy to effectively address congestion is to reduce the demand/supply ratio of the transport infrastructure. Therefore, congestion and queue management can be realized also from a demand-regulation perspective. Metering is one of the measures to control access to a shared infrastructure and an action at this level can ease the flow fluidification in a congested road section [7][8][9]. Speed advisory or variable speed limits proved to be also very effective to alleviate congestion and queues.

The idea of variable speed limits as control action to resolve traffic congestion and improve performances, has been traditionally applied to highway traffic networks. Most of the works dealing with this problem employ macroscopic models

G. De Nunzio, Ph.D. Candidate at GIPSA-Lab and IFPen, France, giovanni.de-nunzio@ifpen.fr

C. Canudas de Wit, Director of Research at CNRS, GIPSA-Lab, NeCS team, Grenoble, France, carlos.canudas-de-wit@gipsa-lab.fr

P. Moulin, Department of Control Signal and Systems at IFPen, RueilMalmaison, France,philippe.moulin@ifpen.fr (namely the METANET model) for their simplicity in a predictive control framework, trying to minimize an objective function. In [10][11] the problem of the propagation of the shock waves is addressed, due to their impact on travel times, fluidity of traffic flows, and safety. By applying a continuous speed control, the authors prove to dilute the shock wave while minimizing the total time spent (TTS) by the vehicles in the network. Furthermore, for a better match between the computed optimal velocity profile and the actual speed advisory, a discretized speed control was also evaluated and further investigated in [12]. A different modeling approach was introduced in [13] and the Variable Length Model (VLM), still applied to highway traffic networks, proved to be effective in describing traffic conditions by decomposing the road section into two cells (a free and a congested cell) of varying size, as a way to monitor the congestion level. This model was also used in a variable speed limits context to contain the growth of the congested area of the road.

Speed advisory for the urban environment was proposed in the '80s [14][15] as a very efficient demand-side queue management strategy and as a pioneer for the modern Advanced Driver Assistance Systems (ADAS). The rather simple initial idea of placing a roadside sign upstream an intersection, indicating the speed to keep to catch a green light, nowadays can be substantially improved thanks to the road communication networks (VANETs). Information on the traffic lights timings will be available through V2V/V2I communication far before the intersections, and an optimal speed advisory can be offered to the drivers and reward the compliant ones with a green wave and more efficient traffic.

In a previous work [1], the authors have investigated the benefits of full information about traffic signals timings and traffic conditions on some global performance metrics. The VLM was adapted and extended to the urban environment (traffic lights regulating vehicular flows) in a variable speed limits control framework, in order to find a compromise between energy consumption and total travel time minimization. An optimal speed was found and analysis showed that a reduction of the speed limit in the cities leads to significant performance improvement. At macroscopic level, reduction of the traveling speed on a road section may translate into a shorter idling time at a signalized intersection, a fluidification of the flow and the speed profile, along with a lower level of pollutant emissions [16].

In this work, the steady-state analysis of the system in [1] has been further developed. Under the hypothesis of equal boundary flows, the existence of a steady-state and an equilibrium point within the domain of the state variables is guaranteed. With respect to the previous work, the perfor- 
mance metrics have been explicitly formulated as functions of the control variable (the speed in the free cell), allowing for a faster solution of the optimization. An assessment of the cost associated with every feasible equilibrium of the system has led also to a performance map of the traffic conditions, which may be seen as an "energy fundamental diagram". Then an exhaustive analysis of the controllability and stability properties of the system has been carried out, proving that the system can be controlled to ensure a faster convergence to the optimal equilibrium, simulating the behavior of the compliant drivers to the speed advisory. Finally a feedback controller was designed to track the optimal equilibrium in the case of equal boundary flows, while a control algorithm was proposed to deal with a varying demand upstream the section. The feedback controller, in both scenarios, proves to outperform the feedforward controller proposed in previous work, as well as the typical city speed limit.

In section II the model and the performance metrics are briefly introduced, in section III an analysis of the system's stability and equilibrium properties is carried out, the controller design is described in section IV and its performance is shown through simulations in section $\mathrm{V}$, conclusion and future perspectives in section VI.

\section{MAThematical FRAMEWORK}

\section{A. Variable Length Model for Urban Networks}

The Variable Length Model, originally proposed for highway traffic [13][17], has been extended to the urban traffic in a variable speed limits control framework [1]. As documented in a previous work, the model depicts traffic lights by means of a switching variable $\alpha(t)$ which describes the phases in the cycle time, and it also allows for the possibility of controlling traffic by acting on the travel speed in the free cell of the section $v_{f}$, in a variable speed limits context.

$$
\left\{\begin{array}{l}
\dot{\rho}_{f}=\left[\varphi_{\mathrm{in}, \alpha}\left(t, v_{f}\right)-\rho_{f} v_{f}\right] \frac{1}{L-l} \\
\dot{\rho}_{c}=\left[w\left(\rho_{m}-\rho_{c}\right)-\varphi_{\text {out }, \alpha}\left(t, v_{f}\right)\right] \frac{1}{l} \\
i=\frac{\rho_{f} v_{f}-w\left(\rho_{m}-\rho_{c}\right)}{\rho_{c}-\rho_{f}}
\end{array}\right.
$$

The periodic nature of the traffic lights, assuming fixedtime cycles, led to the application of the averaging theory [18] on the discrete time-dependent variable $\alpha(t)$, and the simplification of the model to a continuous time one, as follows:

$$
\left\{\begin{array}{l}
\dot{\rho}_{f}=\left[\bar{\varphi}_{\text {in }}\left(v_{f}\right)-\rho_{f} v_{f}\right] \frac{1}{L-l} \\
\dot{\rho}_{c}=\left[w\left(\rho_{m}-\rho_{c}\right)-\bar{\varphi}_{\text {out }}\left(v_{f}\right)\right] \frac{1}{l} \\
i=\frac{\rho_{f} v_{f}-w\left(\rho_{m}-\rho_{c}\right)}{\rho_{c}-\rho_{f}}
\end{array}\right.
$$

where it is reminded that the state variables represent the densities in the free and in the congested cell of the section $\left(\rho_{f}, \rho_{c}\right)$, and the length of queue of congested vehicles $(l)$. The control variable is $v_{f}$. The macroscopic parameters $w$, $\rho_{m}$ and $L$ represent the back-propagation speed of the congestion, the jam density and the section length, respectively. The boundary flows traversing the traffic lights are averaged over the signals cycle time, with $\bar{\alpha}$ being the signal split ratio, and are defined as:

$$
\begin{aligned}
& \bar{\varphi}_{\text {in }}\left(v_{f}\right)=\bar{\alpha}_{i-1} \cdot \min \left\{D_{\text {in }}, S_{f}\right\} \\
& \bar{\varphi}_{\text {out }}\left(v_{f}\right)=\bar{\alpha} \cdot \min \left\{D_{c}, S_{\text {out }}\right\}
\end{aligned}
$$

In the case of inequality of the boundary flows, the system converges naturally to a fully free or a fully congested state. These cases, for the time being, are not of primary importance in the eco-driving benefits evaluation, because if the section is fully congested the control authority is lost, if the section is fully free the following analysis still holds.

Therefore, the existence of an equilibrium level of congestion within the section limits represents a more interesting context of investigation. Assuming that the traffic lights at the two ends are equal, and that there is enough demand upstream of the section and enough supply downstream, the boundary flows are only determined by the maximum value of the demand/supply functions depending on the speed limit in the section:

$$
\bar{\varphi}_{\mathrm{in}}\left(v_{f}\right)=\bar{\varphi}_{\mathrm{out}}\left(v_{f}\right)=\varphi_{m}\left(v_{f}\right)=\bar{\alpha} \frac{w \rho_{m}}{v_{f}+w} v_{f}
$$

Thus, the system may be rewritten as:

$$
\left\{\begin{array}{l}
\dot{\rho}_{f}=\left[\bar{\alpha} \frac{w \rho_{m}}{v_{f}+w} v_{f}-\rho_{f} v_{f}\right] \frac{1}{L-l} \\
\dot{\rho}_{c}=\left[w\left(\rho_{m}-\rho_{c}\right)-\bar{\alpha} \frac{w \rho_{m}}{v_{f}+w} v_{f}\right] \frac{1}{l} \\
\dot{l}=\frac{\rho_{f} v_{f}-w\left(\rho_{m}-\rho_{c}\right)}{\rho_{c}-\rho_{f}}
\end{array}\right.
$$

\section{B. Traffic Performance Metrics}

Traffic conditions evaluation is performed at steady-state and different performance metrics have been tailored to the employed model [1]. In this work the adopted metrics will be formulated at the equilibrium as functions of the sole control variable $v_{f}$, in order to make clearer the dependence of the traffic performance on the speed limits. Under the assumption of equal boundary flows, given a set of initial conditions $\left(\rho_{f}^{0}, \rho_{c}^{0}, l^{0}\right)$, the system (5) converges to the equilibrium:

$$
\begin{aligned}
& \rho_{f}^{*}=\bar{\alpha} \frac{w \rho_{m}}{v_{f}+w} \\
& \rho_{c}^{*}=\rho_{m}-\bar{\alpha} \frac{v_{f} \rho_{m}}{v_{f}+w} \\
& l^{*}=\frac{N_{0}\left(v_{f}+w\right)-\bar{\alpha} \rho_{m} w L}{\rho_{m}\left(v_{f}+w\right)(1-\bar{\alpha})}
\end{aligned}
$$

where $N_{0}$, the time invariant number of vehicles, is:

$$
N_{0}=N=\rho_{f}^{0}\left(L-l^{0}\right)+\rho_{c}^{0} l^{0}
$$

The Instantaneous Travel Time (ITT) is then defined as:

$$
\operatorname{ITT}\left(v_{f}\right)=\frac{L-l}{v_{f}}+\frac{l}{v_{c}}=\frac{N\left(v_{f}+w\right)}{\bar{\alpha} \rho_{m} v_{f} w}
$$


The Total Travel Distance (TTD), calculated over a traffic light cycle $\left(T_{\text {cycle }}\right)$, is:

$$
\operatorname{TTD}\left(v_{f}\right)=v_{f} \bar{\alpha} \frac{w \rho_{m}}{v_{f}+w} L \cdot T_{\text {cycle }}
$$

Finally, the macroscopic energy consumption is obtained as a function of $v_{f}$.

$$
E\left(v_{f}\right)=E_{f}+E_{c}+E_{f \rightarrow c}+E_{c \rightarrow f}
$$

For brevity the entire expression is not reported here, but details on its formulation are reported in previous work [1].

The Total Travel Time (TTT) is of no interest in this particular framework, because it is reminded that the number of vehicles in the section is time invariant.

In this paper the expression of the performance metrics as a function of the control variable $v_{f}$, allows a faster numerical solution of the optimization problem:

$$
\begin{array}{ll}
v_{f}^{*}=\underset{v_{f}}{\operatorname{argmin}} & \left\{E+\sigma_{1} \text { ITT }-\sigma_{2} \text { TTD }\right\} \\
\text { s.t. } & v_{\min } \leq v_{f} \leq v_{\max }
\end{array}
$$

\section{System's Stability Properties}

Given some initial traffic conditions in a section, it is possible to solve the optimization problem (11) and calculate the optimal equilibrium of the system by substituting $v_{f}^{*}$ into (6). Once this information is available, it is natural to design a controller to drive faster the system to the optimal conditions while tracking the optimal speed limit.

The study of the controllabilty of the system is required. However linearization of system (5) yields a non-controllable linear system; one eigenvalue lies on the imaginary axis, and therefore, linearization fails to prove the stability properties of the original nonlinear system at the equilibrium.

The well known definition of stability of an equilibrium point does not hold for system (5). However the system does not diverge, since the boundary flows are equal. In particular, the trajectories of the dynamical system that start near an equilibrium point $\left(\rho_{f}^{*}, \rho_{c}^{*}, l^{*}\right)$ converge to an always different equilibrium, depending on the initial conditions $\left(\rho_{f}^{0}, \rho_{c}^{0}, l^{0}\right)$. An invariance property of the system can be stated as:

Property 1: For a choice of $v_{f}=v_{f}^{*}$, and the initial state $\left(\rho_{f}^{0}, \rho_{c}^{0}, l^{0}\right)$, the system will converge to the equilibrium point $\left(\rho_{f}^{*}, \rho_{c}^{*}, l^{*}\right)$ defined in (6).

As a consequence of this property, starting from arbitrary initial conditions will make impossible to reach an equilibrium $\left(\rho_{f}^{*}, \rho_{c}^{*}, l^{*}\right)$ associated to different initial conditions while conserving also the speed $v_{f}^{*}$ associated to it. In other words, given a certain initial traffic condition and calculated an optimal speed limit $v_{f}^{*}$, it is possible to track only the equilibrium (6) while conserving the optimal speed.

This dependence on the initial conditions, that affects the canonical definition of stability of the equilibrium, may be overcome by observing that $N_{0}$ appears only in the expression of $l^{*}$. The length of congestion can be written as a function of the two densities $\rho_{f}$ and $\rho_{c}$ as follows:

$$
l=\frac{N-\rho_{f} L}{\rho_{c}-\rho_{f}}
$$

and a state transformation from $l$ to $N$ will allow system (2) to be equivalently written as:

$$
\left\{\begin{array}{l}
\dot{\rho}_{f}=\left[\bar{\varphi}_{\text {in }}-\rho_{f} v_{f}\right] \frac{\rho_{c}-\rho_{f}}{\rho_{c} L-N} \\
\dot{\rho}_{c}=\left[w\left(\rho_{m}-\rho_{c}\right)-\bar{\varphi}_{\text {out }}\right] \frac{\rho_{c}-\rho_{f}}{N-\rho_{f} L} \\
\dot{N}=\bar{\varphi}_{\text {in }}-\bar{\varphi}_{\text {out }}
\end{array}\right.
$$

Under the working hypothesis of equal boundary flows (4) and the consequence that the number of vehicles is time invariant, system (5) is then described by only two dynamic equations:

$$
\left\{\begin{aligned}
\dot{\rho}_{f} & =\left[\bar{\alpha} \frac{w \rho_{m}}{v_{f}+w} v_{f}-\rho_{f} v_{f}\right] \frac{\rho_{c}-\rho_{f}}{\rho_{c} L-N} \\
\dot{\rho}_{c} & =\left[w\left(\rho_{m}-\rho_{c}\right)-\bar{\alpha} \frac{w \rho_{m}}{v_{f}+w} v_{f}\right] \frac{\rho_{c}-\rho_{f}}{N-\rho_{f} L}
\end{aligned}\right.
$$

given $N(t)=N_{0}$ for all $t>0$, which now may be seen as a system parameter.

However the parameter $N$ has a well defined domain of existence, in order to ensure that the length of the congested cell $(l)$ at steady-state does not exceed the boundaries of the section $(0<l<L)$. Therefore, by imposing $0<l^{*}<L$, it follows that:

$$
\rho_{m} L \frac{\bar{\alpha} w}{v_{f}+w}<N<\rho_{m} L\left[(1-\bar{\alpha})+\frac{\bar{\alpha} w}{v_{f}+w}\right]
$$

which can be also written as:

$$
\rho_{f}^{*} L<N<\rho_{c}^{*} L
$$

Then, a condition has to be respected also by the initial conditions for the densities in the two cells:

$$
\rho_{f}^{0} L<N<\rho_{c}^{0} L
$$

Intuitively, it is not possible to have a high (low) number of vehicles if we set low (high) initial densities in the two cells.

After this important simplification step, a theorem for stability of system (14) can be given:

Theorem 1: The equilibrium point $\left(\rho_{f}^{*}, \rho_{c}^{*}\right)$ is asymptotically stable if and only if:

- $N$ satisfies (16);

- the initial conditions $\left(\rho_{f}^{0}, \rho_{c}^{0}\right)$ satisfy (17).

Proof: Let the state of system (14) be $x=\left[\rho_{f}, \rho_{c}\right]$ and the input be $u=v_{f}$. The error system evaluated at the equilibrium is of the form:

$$
\dot{x}=\left.A\right|_{\left(x^{*}, u^{*}\right)} e+\left.B\right|_{\left(x^{*}, u^{*}\right)} \tilde{u}
$$

with $e=x-x^{*}$ and $\tilde{u}=u-u^{*}$. The matrices $A$ and $B$ are:

$$
\begin{aligned}
& A=\left[\begin{array}{cc}
-\frac{v_{f}\left(\rho_{c}-\rho_{f}\right)}{\rho_{c} L-N} & 0 \\
0 & -\frac{w\left(\rho_{c}-\rho_{f}\right)}{N-\rho_{f} L}
\end{array}\right] \\
& B=\left[\begin{array}{c}
\frac{\left(\rho_{c}-\rho_{f}\right)\left(-\bar{\alpha} \rho_{m} w^{2}+\rho_{f}\left(v_{f}+w\right)^{2}\right)}{\left(N-\rho_{c} L\right)\left(v_{f}+w\right)^{2}} \\
\frac{-\bar{\alpha}\left(\rho_{c}-\rho_{f}\right) \rho_{m} w^{2}}{\left(N-\rho_{f} L\right)\left(v_{f}+w\right)^{2}}
\end{array}\right]
\end{aligned}
$$




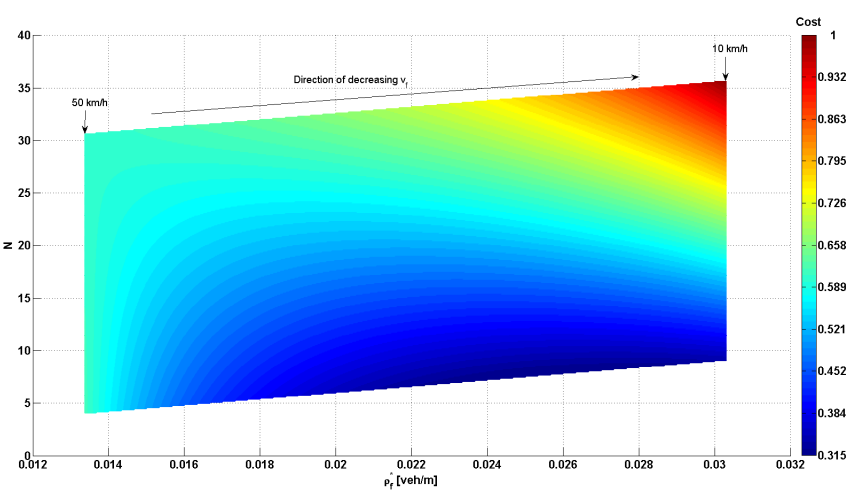

Fig. 1: Energy fundamental diagram. Graph of all the feasible equilibrium points of the system.

The Variable Length Model, by construction, ensures that $\rho_{c}>\rho_{f}$ [13]. The control variable is positive and is subject to the constraints $v_{\min } \leq v_{f} \leq v_{\max }$. Thus, the negativity of two eigenvalues, calculated at the equilibrium, is ensured by condition (16).

Asymptotic stability of the linearized system proves the asymptotic stability of the reduced nonlinear system (14) at any feasible equilibrium point.

Feasibility of the equilibria is ensured by condition (16) on $N$ together with condition (17) on the initial conditions, while respecting also the city speed limits. In order to study the properties of the equilibria, a graph of all the feasible equilibrium points was obtained in Fig.1. Besides the equilibrium density in the free cell $\rho_{f}^{*}$ and the number of vehicles $N$, the third dimension of the graph, in changing colors, is represented by the cost at steady-state associated with each equilibrium. The cost is calculated as in (11) for a choice of $\left(\sigma_{1}, \sigma_{2}\right)=(1.2,0.2)$, after normalization of the terms of the objective function with respect to their maximum value for $v_{f} \in\left[v_{\min }, v_{\max }\right]$. The graph may be seen as an energy fundamental diagram in which a performance index is associated to each equilibrium density. From (6) it is evident that the equilibrium densities do not depend on $N$, therefore for a fixed value of $v_{f}$ the equilibrium densities are constant. In the graph the equilibrium density $\rho_{f}^{*}$ moves from the left to the right as $v_{f}$ decreases. The cost associated to each equilibrium can vary quite significantly. Our control variable $v_{f}$ can have an important impact on the traffic efficiency and it appears clearly how low speeds can severely increase the total cost, due to the impact on the ITT. Intuition suggests that for each value of $N$ there is an optimal value of $v_{f}$ and consequently an optimal equilibrium $\left(\rho_{f}^{*}, \rho_{c}^{*}\right)$ that minimizes the objective function locally.

The optimization problem can be reformulated as follows:

$$
\begin{array}{ll} 
& v_{f}^{*}=\underset{v_{f}}{\operatorname{argmin}}\left\{E+\sigma_{1} \mathrm{ITT}-\sigma_{2} \mathrm{TTD}\right\} \\
\text { s.t. } & v_{\min } \leq v_{f} \leq v_{\max } \\
& \rho_{f}^{*} L<N<\rho_{c}^{*} L \\
& \rho_{f}^{0} L<N<\rho_{c}^{0} L
\end{array}
$$

\section{Control Design}

Being the system asymptotically stable, a controller would not serve stabilization purposes, but, in absence of external disturbances, it would just speed up the convergence of the system to the calculated optimal equilibrium point. The control action may be seen as the compliant drivers' response to the optimal speed advisory.

Given the linear system described by the matrices (19), a Linear Quadratic Regulator (LQR) is designed to track the optimal equilibrium $\left(x^{*}, u^{*}\right)=\left(\rho_{f}^{*}, \rho_{c}^{*}, v_{f}^{*}\right)$. The control law is of the form:

$$
u=u^{*}-K\left(x-x^{*}\right)
$$

where $\tilde{u}=-K e=-K\left(x-x^{*}\right)$, and the gain $K$ is obtained through the LQ design.

For the choice of the weighting matrices $Q$ and $R$ in the objective function of the LQR design, a specific characteristic of the eigenvalues of the reduced linear system was utilized. The real part of the eigenvalues of the linearized system becomes more or less negative depending on the initial conditions and the choice of the parameter $N$. In particular, roughly speaking, if $N$ is high, the mode associated to $\rho_{f}$ is more stable than the one of $\rho_{c}$, and viceversa.

Therefore, in the design of the weighting matrices $Q$ and $R$ of the LQ tracker, a sort of gain scheduling approach was used, in order to weight more the less stable mode:

$$
\begin{aligned}
Q & =\left[\begin{array}{cc}
2000\left(1-\frac{N}{\rho_{m} L}\right) & 0 \\
0 & 2000 \frac{N}{\rho_{m} L}
\end{array}\right] \\
R & =0.00005
\end{aligned}
$$

\section{Simulation Results}

The simulation scenario under analysis presents one road section of length $\mathrm{L}$ with two traffic lights at the two ends regulating inflow and outflow. The traffic lights are modeled by the continuous variables $\bar{\alpha}_{1,2}$. The section is divided into two cells according to the model dynamics and one macroscopic fundamental diagram is defined by the parameters $w$, $\rho_{m}$ and the control input $v_{f}$. Two sets of simulations, with the controller being applied on the general system (2), aim at showing the effectiveness of the feedback controller both in the case of equal boundary flows and in the case of changing upstream demand.

TABLE I: Simulation parameters

\begin{tabular}{c|c|c|c}
\hline Parameter & Description & Value & Unit \\
\hline$L$ & section length & 300 & $\mathrm{~m}$ \\
$w$ & congestion speed & 21.6 & $\mathrm{~km} / \mathrm{h}$ \\
$\rho_{m}$ & jam density & 133 & $\mathrm{veh} / \mathrm{km}$ \\
$v_{\min }$ & minimum speed limit & 10 & $\mathrm{~km} / \mathrm{h}$ \\
$v_{\max }$ & maximum speed limit & 50 & $\mathrm{~km} / \mathrm{h}$ \\
$\rho_{f}^{0}$ & initial free-cell density & 10 & $\mathrm{veh} / \mathrm{km}$ \\
$\rho_{c}^{0}$ & initial congested-cell density & 120 & $\mathrm{veh} / \mathrm{km}$ \\
$l^{0}$ & initial congestion length & 200 & $\mathrm{~m}$ \\
$N_{0}$ & initial number of vehicles & 25 & \\
\hline
\end{tabular}




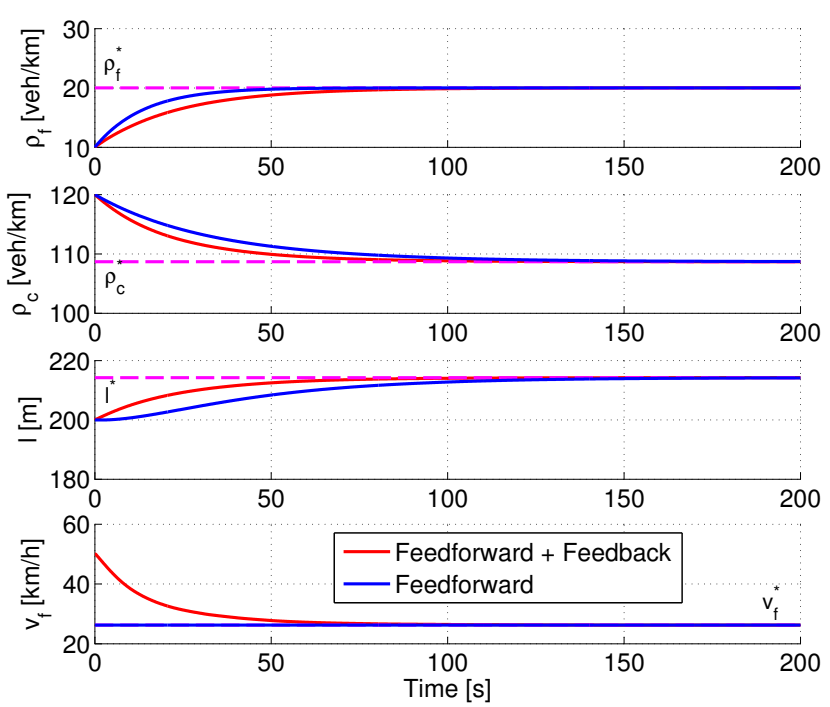

Fig. 2: Equal boundary flows - State dynamics of the nonlinear system and applied control input with and without LQ feedback control action.

\section{A. Equal boundary flows}

The first simulation set is conducted with equally timed traffic lights at the two ends of the section. Under the made assumptions, model (14) holds. The optimal velocity $v_{f}^{*}$ is calculated based on the initial conditions, corresponding to an initial number of vehicles $N_{0}=N=25$. The behavior of the system, with just the feedforward action $v_{f}=v_{f}^{*}$, has already been shown in [1]. Here the feedback controller is able to drive faster the system to the optimal equilibrium $\rho_{f}^{*}, \rho_{c}^{*}, l^{*}, v_{f}^{*}$, as shown in Fig.2. In particular the congestion length settles at the equilibrium in a shorter time: the rise time (10-90\%) with the feedback control is of 51.4 seconds, as opposed to 85.8 seconds with the only feedforward action, meaning a reduction of $40 \%$. At steady-state there are no differences between the two responses of the system and, evidently, the performance metrics on a cycle time of the traffic lights at the equilibrium would give the same value. The transient of the system, however, has been improved and the feedback action helps to give an insight into the desired response of the compliant drivers to the speed advisory.

\section{B. Varying upstream demand}

In the case of a varying demand, assumption on the time invariance of the number of vehicles does not hold and system (14) is not sufficient anymore to describe the state dynamics. Unbalance of the boundary flows will lead the system to converge to a fully-congested or a fully-free state.

The previous analysis on the optimality of the feasible and stable equilibrium points cannot be applied directly to this case, due to the lack of analytic expressions fully describing the equilibria $\left(\rho_{f}^{*}, \rho_{c}^{*}, l^{*}\right)$. However, although nothing can be stated about the convergence value of $l^{*}$, the densities preserve a well defined equilibrium depending on $\bar{\alpha}_{1,2}$ but
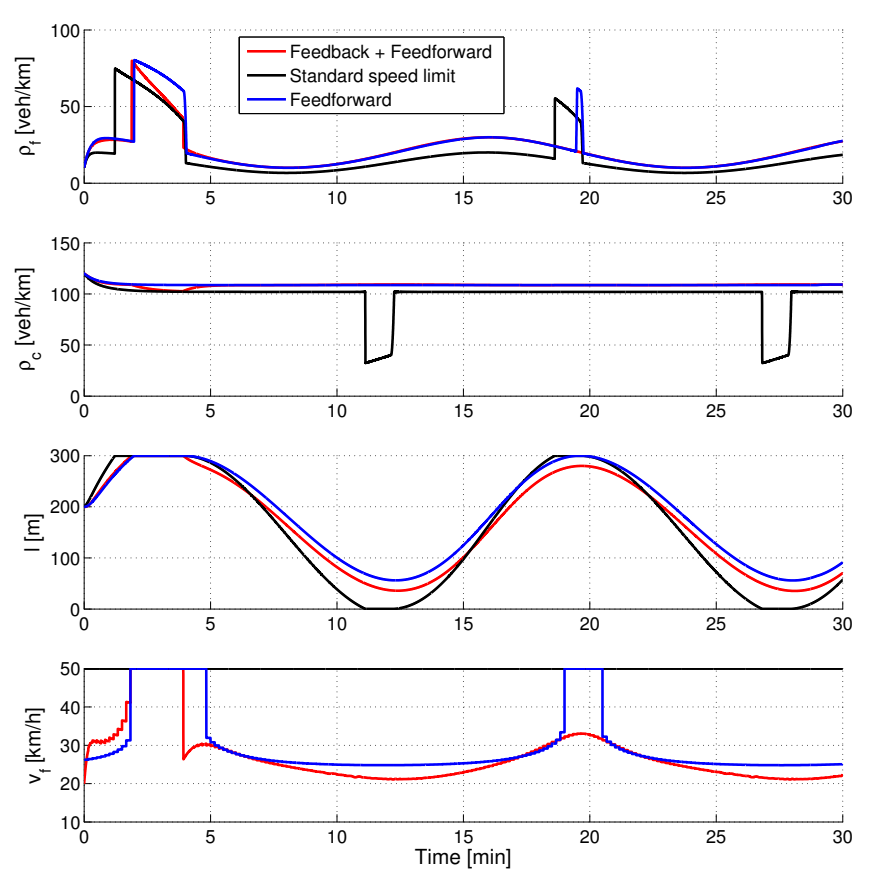

Fig. 3: Varying upstream demand - The proposed control strategy (in red) is compared to the simpler feedforward controller $v_{f}=v_{f}^{*}$ (in blue), and to the standard case with the maximum speed limit $v_{f}=50 \mathrm{~km} / \mathrm{h}$ (in black).

unaffected by the varying number of vehicles:

$$
\begin{aligned}
& \rho_{f}^{*}=\bar{\alpha}_{1} \frac{w \rho_{m}}{v_{f}+w} \\
& \rho_{c}^{*}=\rho_{m}-\bar{\alpha}_{2} \frac{v_{f} \rho_{m}}{v_{f}+w}
\end{aligned}
$$

Provided that no optimality is guaranteed in this scenario, the control strategy could be adapted to the case of time varying boundary flows by following a simple algorithm:

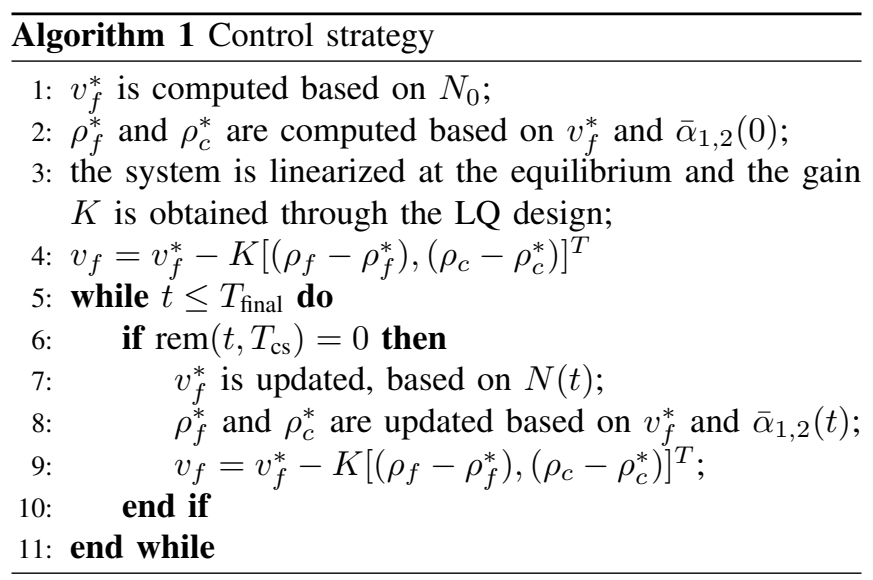

At time $t=0$, the reference speed $v_{f}^{*}$ and the associated equilibrium densities $\left(\rho_{f}^{*}, \rho_{c}^{*}\right)$ are computed as in the case of equal boundary flows, assuming that there will be no variation of $N$. This will be the initial reference to be tracked by the controller, and an update will occur every $T_{\mathrm{cs}}=10 \mathrm{~s}$.

Simulations proved that this control strategy is beneficial in the case of varying upstream demand and the feedback 
action is able to provide performance improvement as compared to both the simpler feedforward controller $v_{f}=v_{f}^{*}$, and the case of a standard city speed limit of $50 \mathrm{~km} / \mathrm{h}$. The test was conducted with the following values of $\bar{\alpha}_{1,2}$ :

$$
\bar{\alpha}_{1}=\bar{\alpha}_{2}+\frac{1}{6} \cos (t / 150) ; \quad \bar{\alpha}_{2}=\frac{1}{3}
$$

Starting from the chosen initial conditions, being $\bar{\alpha}_{1}$ initially higher than $\bar{\alpha}_{2}$, the section quickly saturates in all scenarios. At saturation, the two cells are treated as fixedlength cells, as in the CTM, and no longer necessarily represent the free and congested cells. Saturation actually occurs earlier in the standard-speed-limit case, due to the constant higher speed $v_{f}=50 \mathrm{~km} / \mathrm{h}$. However the feedback controller tries to approach the reference equilibrium by increasing the speed and letting vehicles out of the section at the maximum allowed flow. When the inflow becomes smaller than the outflow $\left(\bar{\alpha}_{1}<\bar{\alpha}_{2}\right)$, the proposed feedback control strategy leaves the fully-congested state before the other scenarios. The feedback controller is able to relieve the congestion much more effectively than the other two policies, in particular at $t \simeq 20$ minutes saturation is avoided.

The performance evaluation here is carried out by calculating the value of the following cost functional:

$$
J=E+\sigma_{1} \mathrm{ITT}-\sigma_{2} \mathrm{TTD}+\sigma_{3} \mathrm{TTT}
$$

The cost function has been slightly modified with respect to the one previously introduced, because in this scenario the number of vehicles is time-varying and the Total Travel Time (TTT) is an important metric to be taken into account.

The results for each performance metric are reported here:

\begin{tabular}{|c|c|c|c|c|}
\hline & E & ITT & TTD & TTT \\
\hline $\begin{array}{c}\text { Proposed control strategy vs. } \\
\text { Standard speed limit }\end{array}$ & $-40 \%$ & $+44 \%$ & $-23 \%$ & $+14 \%$ \\
\hline $\begin{array}{c}\text { Proposed control strategy vs. } \\
\text { Feedforward }\end{array}$ & $-3 \%$ & $-8 \%$ & $-2 \%$ & $-7 \%$ \\
\hline
\end{tabular}

The proposed control strategy outperforms the standard speed limit in the energy consumption but loses performance in the other three metrics. As compared to the feedforward control $v_{f}=v_{f}^{*}$, the proposed strategy improves the performance in all the metrics, except a small loss in the TTD.

In order to evaluate the overall cost, the choice of the new weights is $\left(\sigma_{1}, \sigma_{2}, \sigma_{3}\right)=(0.6,0.2,0.6)$, after normalization of the terms of the cost functional. Clearly the choice of the weighting factors influences the trade-off and the overall performance gain. However, even with the current choice of weights which gives more importance to the travel time metrics together than to the energy consumption, the proposed control strategy enables remarkable improvements. The comparison of the overall cost of the strategies follows:

\begin{tabular}{|c|c|}
\hline Proposed control strategy vs. Standard speed limit & $-14 \%$ \\
\hline Proposed control strategy vs. Feedforward & $-6 \%$ \\
\hline
\end{tabular}

\section{CONCLUSions}

The steady-state analysis of the Urban Variable Length Model has been extended with a stability analysis of the equilibrium points. Controllability of the nonlinear system has been studied and an LQ feedback controller was designed on the linearized system. The controller was tested in simulation initially in a scenario with equal boundary flows to assess its capability of driving the system to the desired optimal equilibrium. Then a control algorithm was proposed to respond to a varying upstream demand. The feedback controller was able to improve the overall performance as compared to the standard city speed limit and the feedforward control action.

Future research will aim at the validation in a microscopic simulator of the proposed control strategy as well as the comparison of the performance metrics between the macroscopic and the microscopic simulation environment. Evaluation of sections concatenation effects and analysis of more complex networks remain also primary objectives.

\section{REFERENCES}

[1] G. De Nunzio, C. Canudas de Wit, and P. Moulin, "Urban Traffic Eco-driving : a macroscopic steady-state analysis," in IEEE European Control Conference, 2014.

[2] E. Brockfeld, R. Barlovic, A. Schadschneider, and M. Schreckenberg, "Optimizing traffic lights in a cellular automaton model for city traffic," Physical Review E, vol. 64, 2001.

[3] K. Aboudolas, M. Papageorgiou, and E. Kosmatopoulos, "Store-andforward based methods for the signal control problem in large-scale congested urban road networks," Transportation Research Part C, vol. 17, pp. 163-174, 2009.

[4] A. Barisone, D. Giglio, R. Minciardi, and R. Poggi, "A macroscopic traffic model for real-time optimization of signalized urban areas," in IEEE Conference on Decision and Control, 2002.

[5] J. Haddad, B. De Schutter, D. Mahalel, I. Ioslovich, and P.-O. Gutman, "Optimal steady-state control for isolated traffic intersections," IEEE Transactions on Automatic Control, vol. 55, no. 11, 2010.

[6] S. Lin, B. De Schutter, Y. Xi, and H. Hellendoorn, "Efficient networkwide model-based predictive control for urban traffic networks," Transportation Research Part C, vol. 24, pp. 122-140, 2012.

[7] M. Papageorgiou, H. Haj-Salem, and J. M. Blosseville, "ALINEA: A local feedback control law for on-ramp metering," Transportation Research Record, no. 1320, 1991.

[8] C. F. Daganzo, "Urban gridlock: Macroscopic modeling and mitigation approaches," Transportation Research Part B, vol. 41, no. 1, 2007.

[9] N. Groot, B. De Schutter, and H. Hellendoorn, "Integrated Model Predictive Traffic and Emission Control Using a Piecewise-Affine Approach," IEEE Transactions on Intelligent Transportation Systems, vol. 14 , no. 2, pp. 587-598, 2013.

[10] A. Hegyi, B. De Schutter, and J. Hellendoorn, "Optimal Coordination of Variable Speed Limits to Suppress Shock Waves," IEEE Transactions on Intelligent Transportation Systems, vol. 6, no. 1, 2005.

[11] A. Hegyi, S. P. Hoogendoorn, M. Schreuder, H. Stoelhorst, and F. Viti, "SPECIALIST: A dynamic speed limit control algorithm based on shock wave theory," in IEEE Conference on Intelligent Transportation Systems, 2008.

[12] J. R. D. Frejo, A. Núñez, B. De Schutter, and E. F. Camacho, "Model predictive control for freeway traffic using discrete speed limit signals," in IEEE European Control Conference, 2013.

[13] C. Canudas de Wit, "Best-effort highway traffic congestion control via variable speed limits," in IEEE Conference on Decision and Control and European Control Conference, 2011.

[14] R. S. Trayford, B. W. Doughty, and M. J. Wooldridge, "Fuel saving and other benefits of dynamic advisory speeds on a multi-lane arterial road," Transportation Research Part A, vol. 18, no. 5-6, 1984.

[15] R. S. Trayford, B. W. Doughty, and J. W. van der Touw, "Fuel economy investigation of dynamic advisory speeds from an experiment in arterial traffic," Transportation Research Part A, vol. 18, 1984.

[16] J. Archer, N. Fotheringham, M. Symmons, and B. Corben, "The impact of lowering speed limits in urban/metropolitan areas," Monash University Accident Research Center, Australia, Tech. Rep., 2008.

[17] C. Canudas de Wit and A. Ferrara, "A New Variable-length Cell Model for Traffic Systems," http://hal.archives-ouvertes.fr/hal-00966968, Internal, Tech. Rep., 2014.

[18] H. K. Khalil, Nonlinear systems, 3rd ed. Prentice Hall, 2002. 